\title{
Publisher Correction: Topological frustration induces unconventional magnetism in a nanographene
}

Shantanu Mishra, Doreen Beyer, Kristjan Eimre (D), Shawulienu Kezilebieke, Reinhard Berger, Oliver Gröning, Carlo A. Pignedoli, Klaus Müllen, Peter Liljeroth (D), Pascal Ruffieux (), Xinliang Feng and Roman Fasel (D)

Correction to: Nature Nanotechnology https://doi.org/10.1038/s41565-019-0577-9, published online 9 December 2019.

In the version of this Letter originally published online, in Fig. 2e and Fig. 3j, "Spin density Bohr magneton $\left(\mu_{\mathrm{B}}\right)$ " should have been "Spin density $\left(\mu_{\mathrm{B}}\right)$ ", in Fig. $2 \mathrm{~m}$, each pair of angular brackets ' \langle\rangle ' should have been a ket ' |\rangle ', and, in the caption for Extended Data Fig. 1, ' $n_{\uparrow}=n_{\downarrow} 2$ ' should have been ' $n_{\uparrow}=n_{\downarrow}+2$ '. These errors have been corrected in all versions of the Article.

Published online: 17 December 2019

https://doi.org/10.1038/s41565-019-0621-9

๑) The Author(s), under exclusive licence to Springer Nature Limited 2019 